\title{
Raltegravir: Molecular Basis of Its Mechanism of Action
}

\author{
Jean-François Mouscadet and Luba Tchertanov \\ LBPA, CNRS, Ecole Normale Supérieure de Cachan, France
}

\begin{abstract}
Integration of the HIV-1 viral DNA generated by reverse transcription of the RNA genome into the host cell chromosomes is a key step of viral replication, catalyzed by the viral integrase. In October 2007, the first integrase inhibitor, raltegravir, was approved for clinical use under the name of Isentress TM. The results of the various clinical trials that have evaluated raltegravir have been very encouraging with regard to the immunological and virological efficacy and tolerance. However, as observed for other anti-retrovirals, specific resistance mutations have been identified in patients failing to respond to treatment with raltegravir. Although knowledge of the integrase structural biology remains fragmentary, the structures and modeling data available might provide relevant clues on the origin of the emergence of these resistance mutations. In this review, we describe the mechanism of action of this drug and the main data relating to its use in vivo, together with recent structural data important to our understanding of the origin of viral resistance.

Key words: HIV-1 integrase, raltegravir, isentress, resistance, molecular modeling

Abbreviations: $\mathrm{ARV}=$ antiretroviral $\mathrm{CCD}=$ catalytic core domain; hDNA = host DNA; HIV = Human immunodeficiency virus; IN = integrase; INI = integrase inhibitor; INSTI = integrase strand transfer inhibitor; LTR = long terminal repeat; $\mathrm{PIC}=$ preintegration complex; $\mathrm{PR}=$ protease $\mathrm{RT}=$ reverse transcriptase; tDNA $=$ target DNA
\end{abstract}

\section{INTRODUCTION}

HIV replication is driven by a molecular engine consisting of three viral enzymes: reverse transcriptase $(\mathrm{RT})$, protease (PR) and integrase (IN). Integrase catalyzes the covalent insertion of the viral DNA produced by reverse transcription of the RNA into the chromosomes of infected cells. Once integrated, the provirus persists in the host cell and serves as a template for the transcription of viral genes and replication of the viral genome, leading to the production of new viruses. Due to its key function in the viral life cycle, IN is an attractive target for antiretroviral drugs (ARVs) and has thus been the object of intensive pharmacological research over the last 20 years. Since the end of the 1990s, several inhibitors with genuine antiviral activity have been identified and developed. Several of these compounds, including raltegravir (Isentress $^{\mathrm{TM}}$ ) and elvitegravir in particular, have shown great promise, ensuring the rapid recognition of integrase inhibitors (INIs) as an important new class in the arsenal of antiretroviral drugs (ARVs) [42]. Raltegravir was approved for clinical use in October 2007, following the demonstration of a rapid, potent and sustained antiretroviral effect in patients with advanced HIV-1 infection. It is well tolerated and, due to its mechanism of action, is likely to be active against viruses resistant to other class of antiretroviral drugs, such as nucleosides, nucleotides and non nucleosides reverse transcriptase inhibitors, protease and entry inhibitors. However as with other antivirals, resistance mutations, located in the integrase gene of replicating viruses and preventing the establishment of specific interactions between the inhibitor and its integrase target, rapidly emerge associated with a reduced susceptibility to the drug. In this review, we focus on the mechanism of action of raltegravir in vitro and in vivo and we present the structural data that shed light on the molecular basis of its inhibitory potency and on the origin of the emergence of resistance.

\section{INTEGRASE BIOCHEMISTRY}

Catalytic activity. Virological data have demonstrated that the precursor of the integrated genome, or provirus, is the linear viral DNA produced by reverse transcription of the RNA genome [11]. Two reactions are required for the covalent insertion of the viral genome. First, integrase binds to short sequences located at either end of the viral long terminal repeat (LTR) and catalyzes an endonucleolytic cleavage, in a reaction known as 3' processing, removing a dinucleotide at either end of both 3' LTRs, leading to the exposure of a conserved CA sequence. Integration sensu stricto, or strand transfer, then occurs through attack of the phosphodiester backbone in target DNA by the 3' hydroxyl groups of the processed DNA (Fig. 1 A). Strand transfer takes place concomitantly for both extremities, with a five-base gap between insertion points. In vivo, these two reactions are spatially and temporally separated and energetically independent: 3 ' processing takes place in the cytoplasm of infected cells, whereas strand transfer occurs in the nucleus. Both reactions are one-step transesterification reactions with no covalent intermediates between integrase and the DNA [14].

Cellular enzymes are responsible for cleaving the protruding 5' ends of the viral DNA that remain unattached during strand transfer and repairing flanking 
gaps, thereby completing the integration process [10]. The final product is a covalently inserted viral genome, colinear with cellular genes, with a short (e.g. 5 bp) duplication on either side, the length of which is a hallmark of the retrovirus concerned. It is possible to reproduce the whole integration process in vitro, using short DNA fragments or oligonucleotides mimicking the sequence of the ends of the LTR in the presence of recombinant integrase [14]. In terms of specificity, only the terminal 5'CA is strictly required for 3' processing. The mutation of this dinucleotide completely abolishes the reaction, whereas the requirements concerning the adjacent sequences are less stringent [33]. It is intrinsically difficult to demonstrate the specificity of the enzyme for the viral DNA due to its ability to bind specific and non specific DNA sequences simultaneously. Nevertheless, recent advances have led to the development of an assay faithfully reproducing fully concerted integration in vitro [60]. In vitro, a third reaction, known as disintegration, may be observed in which the reverse strand transfer process occurs [21]. Unlike 3' processing and strand transfer, which depend on the integrity of the enzyme, disintegration may be catalyzed by the isolated catalytic core domain containing the active site. There is no experimental evidence to suggest that disintegration occurs in vivo, but pharmacological approaches involving the stabilization of integrase on the strand transfer intermediate might favor this reverse reaction, thereby decreasing the efficiency of integration.

Integrase functions in a multimeric form, as shown by the complementation of inactive proteins observed in virions [31]. Dimers formed at either end of the viral DNA molecule are responsible for 3'-processing activity [41]. Pairs of dimers bring together the two ends of the viral DNA, leading to the formation of a tetramer (a dimer-of-dimers), the active form required for concerted integration [34]. During its catalytic cycle, IN must bind simultaneously to the viral DNA (vDNA) and the target DNA (tDNA). Current knowledge of the organization of this tetramer on the DNA is based exclusively on models constructed from partial structural and biochemical data (see below), which may provide a platform for the rational design of new inhibitors.

Cationic cofactor. All integrase activities strictly require the presence of a metallic cationic cofactor, which is coordinated by two residues of the catalytic triad (D64, D116 and E152 for HIV-1 IN) [3]. The catalytic cation may be either $\mathrm{Mn}^{2+}-$ or $\mathrm{Mg}^{2+}$ in vitro, but $\mathrm{Mg}^{2+}$ is the cofactor required in vivo and $\mathrm{Mg}^{2+}$-dependent activities also reproduce physiological activity more faithfully in vitro. IN displays non specific nuclease activity in the presence of $\mathrm{Mn}^{2+}$, and the $\mathrm{Mg}^{2+}$ enzyme is much less tolerant of sequence variations at the ends of the LTR than the $\mathrm{Mn}^{2+}$ enzyme [33]. Several mutations are known to have no effect on IN activity in $\mathrm{Mn}^{2+}$-dependent assays, whereas they do affect IN activity in $\mathrm{Mg}$-dependent assays. For example, mutations of the HHCC domain known to be detrimental for the virus in vivo alter 3 'processing in vitro in the presence of $\mathrm{Mg}^{2+}$, but not in the presence of $\mathrm{Mn}^{2+}$ [56]. In addition, factors promoting integrase multi- merization, such as $\mathrm{Zn}^{2+}$, also specifically stimulate the $\mathrm{Mg}^{2+}$-dependent activity of the enzyme, consistent with the multimeric nature of the functional enzyme [57]. These differences between cofactor activities have resulted in pharmacological discrepancies, as some early IN inhibitors identified on the basis of $\mathrm{Mn}^{2+}$-dependent assays were not active against the $\mathrm{Mg}^{2+}$ enzyme. Based on a model of another phosphatidyl transferase, the 3'-5' exonuclease of E. coli DNA polymerase I [7], it was suggested early on that the retroviral integrase might contain two metal cation cofactors. The 3D structures of avian sarcoma virus integrase and the Tn5 transposase alone or in complex with DNA have provided structure-based evidence for a two-metal active site structure for retroviral integrases [13, 65]. These considerations eventually led to the incorporation of $\mathrm{Mg}^{2+}$-chelating groups into the rational design of IN inhibitors. Such groups are present in all effective IN inhibitors, including raltegravir [40].

\section{DEVELOPMENT OF INTEGRASE INHIBITORS}

Mechanisms of inbibition. In terms of pharmacological development, two screening strategies have been considered for the development of IN inhibitors: one based on the free, unbound protein and the other on the preformed IN $\bullet$ viral DNA (vDNA) complex. Both approaches were demonstrated to be feasible, with the identification of inhibitors of either 3' processing, blocking the binding of IN to viral DNA, or strand transfer, targeting the IN ${ }^{\mathrm{v} D N A}$ complex. Since the early 1990s, a number of compounds inhibiting one or other of these reactions have been identified in vitro [83]. However, the complex resulting from the association of integrase with viral DNA whether isolated from infected cells as a pre-integration complex (PIC), or reconstituted in vitro, is highly stable, keeping the complex together for long enough after the 3 '-processing reaction for subsequent integration to occur [55]. This complex has an intrinsically slow catalytic activity and does not dissociate after 3 ' processing, limiting multiple turnover [86]. This weak catalytic activity is not detrimental in host cells, because a single integration event is sufficient for overall function, but it makes it difficult to develop competitive inhibitors of free IN. For these reasons, the Merck team lead by Dr D. Hazuda suggested in the mid 1990s that the PIC would be a more suitable target for inhibitors. This hypothesis proved to be correct, particularly given that PIC formation probably occurs within a capsid that is not fully dissociated, thus precluding easy access to free IN [1].

The design of new assays for screening ligands of the IN $\bullet$ vDNA complex eventually led to the identification of the first strand transfer inhibitors, L-731, 988 and L-708, 906 at the turn of the century [43]. These compounds compete with the target DNA by binding to the IN $\bullet$ vDNA complex. They recognize a specific site close to the catalytic triad, which opens following a change in conformation induced by the binding and 3' processing of the viral DNA [32]. The first selective inhibitors of strand transfer to be identified were $\alpha, \gamma$ diketoacids (DKAs) [43]. Such compounds based on the $\beta$-ketoenol fragment efficiently chelate the $\mathrm{Mg}^{2+}$ 


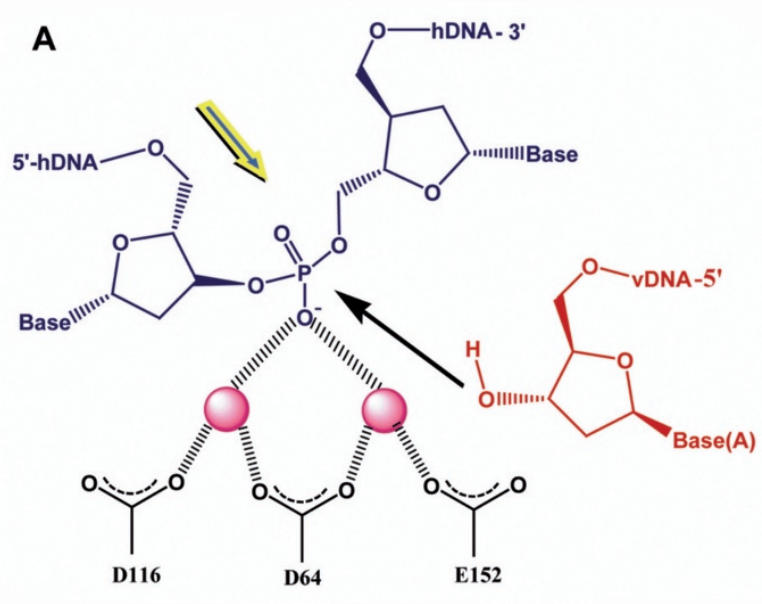

B

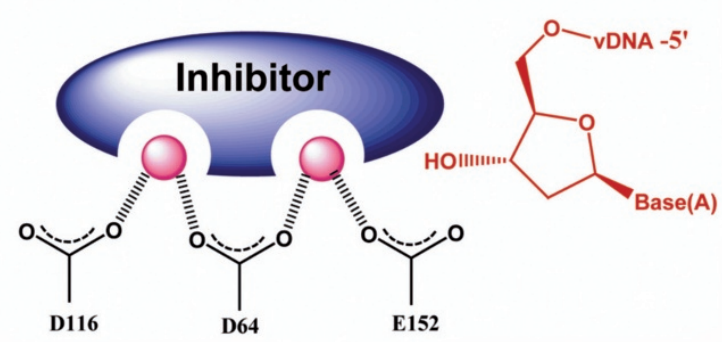

Fig. 1. Strand transfer reaction and proposed mechanism of IN inhibition by INSTIs adapted from (53). A) A trans-esterification reaction involving a nucleophilic attack on the 3 ' hydroxyl group of the two newly processed 3' viral DNA ends on the phosphodiester backbone of the host DNA. The host DNA and viral DNA are shown in blue and red, respectively; the yellow arrow indicates the scissile phosphodiester. B) IN strand transfer inhibitors may chelate the two metal ions in the catalytic site, thereby blocking the binding of host DNA.

cation required for the activity of integrase and their overall affinity for the target depends on their surrounding substituent groups (Fig. 1 B) [53, 90].

The prerequisites for a specific strand transfer inhibitor include the presence of a chemical group in- cluding the heteroatoms, nitrogen or oxygen, capable of binding two divalent cations and a hydrophobic aromatic part of the molecule likely to bind and stabilize the IN $\bullet$ vDNA complex, forming an active pharmacophore responsible for the activity of all strand transfer inhibitors [4, 53] (Fig. 2). Compounds with these properties selectively target and bind to the IN $\bullet$ vDNA complex, close to the $3^{\prime}$ end of the donor DNA, thereby inhibiting target DNA binding, resulting in selective inhibition of the strand transfer reaction with no significant effect on the 3 '-processing reaction [78]. They therefore act as IN•DNA interfacial inhibitors, and are known as integrase strand transfer inhibitors (INSTIs).

The replacement of the carboxylate group by its tetrazolium bioisostere led to the development of 5CITEP (Fig. 2) and its analog, S-1360. Despite the weak activity of these molecules against integrase, the structure of the integrase/5-CITEP complex has been determined, making it possible to construct a model of the structure of the inhibitor pharmacophore bound to the active site metal cation [36]. Modifications to the $\alpha, \gamma$-diketoacid part of the molecule initially led to the replacement of this group by 8-hydroxy quinoline, to increase antiviral activity and to overcome pharmacological limits, such as serum protein binding [98]. Compounds from this family, such as Merck L870, 812 (Fig. 2 ), have potent antiviral activity, providing the proofof-concept for INSTI activity in vivo despite their toxicity in vivo [44]. The L870, 812 series of compounds was not developed further, but the dihydroquinoline JTK303/GS9137 (Fig. 2) derived from quinolone antibiotics was used for further drug development and is now at the advanced clinical development stage, under the name of elvitegravir [80].

Development of raltegravir. The discovery of raltegravir stemmed from investigations of a series of HCV polymerase inhibitors. The architecture of the catalytic site and the arrangement of the metal cations are very similar in integrase and the HCV NS5b RNAdependent RNA polymerase. These similarities led the Merck team to test HCV polymerase inhibitors origi-<smiles>O=C(/C=C(\O)c1nn[nH]n1)c1cc2cc(Cl)ccc2[nH]1</smiles>

5-CITEP<smiles>CN(C)C(=O)C(=O)N(C)c1nc(C(=O)NCc2ccc(F)cc2)c(O)c2ncccc12</smiles>

L-870,812<smiles>COc1cc2c(cc1Cc1cccc(Cl)c1Br)c(=O)c(C(=O)O)cn2[C@@H](CO)C(C)C</smiles>

ELVITEGRAVIR<smiles>Cc1nnc(C(=O)NC(C)(C)c2nc(C(=O)NCc3ccc(F)cc3)c(O)c(=O)n2C)o1</smiles>

RALTEGRAVIR
Fig. 2. Integrase strand transfer inhibitors. 
nally designed as drug-compliant DKA replacements [89]. This led to the identification of a compound with activity in the enzymatic assay, which was further optimized in cell culture $[76,89]$. Raltegravir is a potent inhibitor of the replication of HIV-1 and HIV-2 in vitro $[70,79]$. It is more than 1000 times more selective for integrase than for other phosphatidyl transferases, such as HIV-1 RNAseH and human polymerases. It has an $\mathrm{IC}_{50}$ of 2 to $7 \mathrm{nM}$ for the inhibition of recombinant IN-mediated strand transfer in vitro and an $\mathrm{IC}_{95}$ of 0.019 and $0.031 \mu \mathrm{M}$ in $10 \% \mathrm{FBS}$ and $50 \%$ NHS, respectively, in a cell-based assay [70, 89]. Due to its mode of action, it is independent of HIV-1 tropism (CCR5 and CXCR4) and active against viruses resistant to other classes of antiretroviral drugs, such as nucleoside reverse transcriptase inhibitors, protease inhibitors, fusion and entry inhibitors [39].

\section{Antiviral Potency of Raltegravir}

Antiviral activity in vivo. Phase II and III trials demonstrated a remarkable potency of combinations of raltegravir and other ARVs in treatment-experienced patients $[24,39,88]$. The first phase II assay was a dose-ranging study in patients with documented resistance to at least one drug in each of the three classes of ARVs. This population had considerable experience of treatment and a very high level of drug resistance. There was an approximate $2.0 \mathrm{log}$ copies $/ \mathrm{ml}$ drop in plasma HIV RNA levels by week 24 in the raltegravir group, versus only $0.35 \mathrm{log}$ with optimized therapy alone plus placebo, with no significant difference in viral efficacy between the three dosage groups studied (200, 400, $600 \mathrm{mg}$ ) [39]. For the subsequent doubleblind phase III BENCHMARK I and II studies, in which 699 patients with considerable experience of treatment were enrolled, the combined analysis at 48 weeks showed that $72.3 \%$ and $62.1 \%$ of raltegravirtreated patients had HIV RNA levels of less than 400 and 50 copies $/ \mathrm{ml}$, respectively, whereas such levels were found in only $37.1 \%$ and $32.9 \%$, respectively, of the patients in the placebo group.

The 48-week results recently obtained for the phase III STARTMRK study comparing raltegravir-based and efavirenz-based combination regimens as initial treatment demonstrated that raltegravir suppressed HIV replication more rapidly than efavirenz, this rapid viral decay being of unknown origin [58]. Moreover, preliminary results from a non inferiority study of the use of raltegravir to replace enfuvirtide in patients intolerant to enfuvirtide have shown raltegravir to be virologically effective for sustained periods, with good tolerance for up to 48 weeks. Conversely, the SWITCHMRK 1 and 2 trials, designed to examine the benefit of replacing a protease inhibitor with raltegravir, suggested that the raltegravir combination might not inhibit HIV replication more efficiently. In situations of resistance due to prior treatment failure, switching to raltegravir amounts to monotherapy, with the rapid selection of raltegravir-resistant HIV strains, as the genetic barrier to raltegravir is easily overcome. Nevertheless, these results suggest that raltegravir is an important additional drug for the initial treatment of HIV-1 infection.
Safety. Preclinical studies of toxicity by repeated administration, genotoxicity and toxic effects on development have been conducted with raltegravir, in mice, rats, dogs and rabbits. No mutagenic or teratogenic effect was observed. The effects observed at levels exceeding actual exposure levels revealed no likelihood of a clinical risk in humans [89]. Raltegravir is well tolerated and adverse events are rare. Most frequent drug-related clinical events, such as diarrhea, nausea, headache and fatigue, were moderate and transient [48]. Laboratory abnormalities included an increase in serum lipid, aminotransferase and creatinine concentrations. Increases in creatinine phosphokinase levels, although not statistically significant, led to a cautious recommendation not to use raltegravir concomitantly with other drugs known to increase these levels. In phase II and phase III trials, the frequency of clinical and laboratory adverse events was similar in the raltegravir and placebo groups. In the STARTMRK trial, significantly fewer drug-related clinical adverse events occurred in patients on raltegravir than in those on efavirenz [58]. The BENCHMRK trial suggested a small increase of the risk of cancer in the raltegravir arm, with a relative risk of 1.5, but a recent analysis of all the available data concluded that the relative risk was actually less than 1 [19].

Pharmacokinetics. Raltegravir is administered orally and is rapidly absorbed. Its absolute bioavailability has yet to be determined, but the administration of $400 \mathrm{mg}$ per day results in steady-state levels of the drug in the body within two days, as demonstrated by pharmacokinetics studies. About $83 \%$ of the raltegravir ingested binds to plasma proteins. Animal studies have shown raltegravir penetrate the stomach, liver, small intestine, kidney and bladder effectively, but have suggested that penetration into the brain is limited. Considerable intra- and interindividual variability was observed. Raltegravir is a substrate, but not an inhibitor of P-glycoprotein (Pgp). There is currently no evidence to suggest that inhibitors or inducers of Pgp could affect raltegravir, but this property may affect its absorption [23]. It could also account for the limited diffusion of this drug into the central nervous system. No effect of age or sex has been identified in studies of the pharmacokinetics of raltegravir (no data are available for children) [46]. The half-life of raltegravir in the body is about nine hours, with an initial phase of rapid elimination lasting about 1 hour. At steady state, a slight increase in residual concentrations of the drug is observed, but with no effect on the maximum concentration, making it possible to administer raltegravir twice daily.

Raltegravir is mostly metabolized in the liver, through glucuronidation by uridine diphosphate-glucuronolsy-transferase 1A1 (UGT1A1) to generate a single metabolite, M2. Raltegravir is neither a substrate nor an inhibitor of the cytochrome P450 enzymes, consistent with a lack of interaction with drugs metabolized by P450 isoenzymes, including protease inhibitors. It does not inhibit either UGT1A1 or $2 \mathrm{~B} 7$ and does not induce CYP34A. As raltegravir is mostly metabolized by UGT1A1, it should be used with caution when co-administered with strong inducers of 
UGT1A1, such as rifampicin. This antibiotic has been shown to reduce plasma concentrations of raltegravir, although its impact on the efficacy of raltegravir is unknown. A mutation of the UGT1A1 gene resulting in the production of an inactive enzyme has been identified. Two studies have shown in the concentration of raltegravir to be higher in patients with a homozygous mutant genotype. This genotype seems to be an important factor in interindividual variability, but its clinical relevance, in terms of efficacy and toxicity, is unknown (http://www.emea.europa.edu). Finally, atazanavir, a protease inhibitor affecting glucuronidation, decreases the formation of raltegravir glucuronide and induces a moderate increase in raltegravir concentration $[39,47]$.

Resistance to raltegravir. As with other antiretroviral drugs, resistance to INI emerges through the selection of mutations in the integrase gene affecting the susceptibility of the virus to INI. More than 40 mutations have been specifically associated with resistance to INSTIs in vitro and in vivo [16]. Resistance to raltegravir in vivo has been associated with 14 mutations, to different degrees, but the virologic failure observed during the BENCHMRK trials was unambiguously associated with two principal independent genetic pathways involving primary mutations of residues N155 $(\mathrm{N} 155 \mathrm{H})$ and Q148 (Q148K/R/H) [24, 88]. These mutations were not detected in the various studies on integrase polymorphism in INI-naive patients, confirming their likely role in conferring resistance to this class of drugs. Secondary mutations increasing the fitness of the resistant viruses were identified in both pathways. In particular, the G140S mutation rescues a replication defect resulting from the primary mutation Q148H [27]. Phenotypic analysis showed that the presence of the mutation at position 148 together with one or more secondary mutations resulted in greater resistance to RAL than observed for viruses carrying the mutation $\mathrm{N} 155 \mathrm{H}$. Clonal analysis of the viral populations in 11 patients with treatment failure on raltegravir showed that no viral clone simultaneously carried mutations in position 148 and 155, demonstrating the independence and exclusivity of the two main pathways. Moreover, a switch of resistance profile from residue 155 to residue 148 mutations may occur due to the higher level of resistance to raltegravir conferred by the pathways associated with residue 148 mutation and the greater instability of the pathways associated with residue 155 [68].

A small number of mutations involving residues E92, E157 and Y143 might constitute another pathway of resistance. There is some debate about whether the first two of these mutations are true primary mutations for RAL resistance, whereas the Y143 mutation has been shown to confer a real decrease in susceptibility to the inhibitor [85]. Y143R/C/H mutations occur less frequently and later than the other two mutations [25].

The major IN mutations E92Q, Q148K/R/H, $\mathrm{N} 155 \mathrm{H}$ and E157Q are highly conserved and subject to similar genetic barriers between subtypes $\mathrm{B}$ and CRF02_AG. However, the CRFO2_AG subtype has a stronger genetic barrier to the acquisition of mutations of residue G140 than subtype B [66]. Another showed that treatment failure on raltegravir occurred more rapidly in patients infected with non B subtype viruses, indicating a possible impact of non B-associated polymorphisms on the genetic barrier to raltegravir [85].

\section{Fate of Non Integrated Viral Genomes}

A productive HIV-1 replication in T4 lymphocytes depends on the activation and multiplication of these cells. HIV-1 can enter resting T cells, but in absence of cell activation the fate of the viral genome is uncertain. Replication may abort during the reverse transcription step or be blocked before integration [75, 94, 97]. It has been suggested that incoming HIV-1 subviral complexes may concentrate in the centrosome, in which they may remain in a stable state for several weeks [87]. Thus, HIV-1 may persist in quiescent cells as a longlived, centrosome-associated, preintegration intermediate [95]. Upon cell activation, viral replication may resume, leading to viral gene expression [93] and providing a possible explanation for the unusual decay kinetics of viral load during raltegravir treatment [72]. This may also account for the faster decay kinetics observed with raltegravir than with efavirenz.

In the absence of integration, the linear viral DNA is circularized, probably by non-homologous end joining $[54,59]$ to yield circular forms that do not support viral replication but that might persist in the nucleus for an undetermined period of time [77]. This circularization of viral genomes is in fact one reason for the activity of raltegravir. Indeed, it prevents the genomes from being integrated when the inhibitor, non-covalently bound to the PIC, is eventually released from its binding site. Accordingly, the residence time of raltegravir onto its target was found to be a determinant of its inhibitory potency and is dramatically decreased by the presence of the primary resistance mutations.

In the presence of strand transfer inhibitors, such as raltegravir or elvitegravir, an accumulation of 2-LTR circular forms is observed. The current consensus is that these forms do not play a significant role in viral replication, although non integrated DNA largely exceeds integrated forms in resting $\mathrm{T}$ cells during HAART [22]. However, the production of the viral Nef and Tat proteins has been demonstrated [92] and it has been suggested in various studies that these circular species may be transcribed during HIV-1 infection, so we cannot completely rule out a functional role of these circles in viral replication [12]. In addition, certain integrase mutants unable to mediate integration remain competent for replication in permissive cells, such as CEM MT4 cells, albeit with low efficiency, suggesting the direct involvement of the circles or an integrase-independent integration mechanism based on recombination, for example [73].

In any case, unlike other ARVs, INSTIs do not cause the complete disappearance of the viral genome from infected cells. Instead, they merely prevent genome integration. The fate of the circular species during treatment with INSTIs remains to be determined. A recent study demonstrated that the intensification of raltegravir treatment over a 12 -week period did not decrease low-level plasma viremia in patients on HAART. This finding suggests that residual viremia 
may not result from complete cycles of viral replication including integration.

\section{STRUCTURE-BASED ANALYSis OF INTEGRASE/RALTEGRAVIR INTERACTIONS}

Structural analyses aiming to decipher the determinants of raltegravir binding to integrase should help us to understand the unique mechanism of action of this molecule and facilitate the structure-based design of second-generation inhibitors. Unfortunately, our understanding of the mode of binding of INIs is limited by a lack of knowledge of the structure of the fulllength protein, an accurate description of the binding of the metal cation and experimental structural data about the interaction of IN with its viral and cellular DNA substrates. Neither the structure of isolated fulllength IN nor that of IN in complex with its DNA substrate has yet been determined.

Integrase domains. Integrase is a 288-amino acid protein $(32 \mathrm{kDa})$ encoded by the end of the pol gene. It is produced as part of the Gag-Pol polypeptide precursor, from which it is released by viral protease-mediated cleavage [3]. It has three independent domains [31]: (i) the N-terminal domain (amino acids 1-49), which carries an HHCC motif analogous to a zinc finger, possibly favoring protein multimerization, a key process in integration (2); (ii) the core domain (amino acids 50-212), encompassing the catalytic (D, D35E) motif, also involved in binding the ends of the viral DNA, notably via residue Q148, which is involved in resistance to raltegravir (20); (iii) the C-terminal domain (amino acids 213-288), which binds non specifically to DNA and therefore mostly involved in stabilizing the complex with DNA. The 24 structures available in the Protein data bank describe the three domains separately, or as two-domain fragments consisting of the catalytic core plus the C-terminal domain or the catalytic core plus the N-terminal domain [49].

The published X-ray structures of the catalytic core domain (CCD) include a mutation of the F185 residue introduced to increase the solubility of the enzyme whilst maintaining its catalytic activities in vitro [51]. Crystallization conditions may result in local differences, but the topology of all the structures obtained are similar. The CCD has an $\alpha / \beta$ structure consisting of five $\beta$-sheets and six $\alpha$-helices forming a dimer with two-fold symmetry and a large solvent-excluded interface. Two structures in which the CCD is bound to the $\mathrm{Mg}^{2+}$ cofactor coordinated with the two aspartate residues D64 and D116 have been described [37, 67]. The structures of the isolated $\mathrm{N}$ - and C-terminal domains have been determined by NMR. Dimers of the $\mathrm{N}$-terminal domain have been observed in solution, with each monomer forming a highly $\alpha$-helical structure, with 4 helices stabilized by $\mathrm{Zn}^{2+}$ coordination and hydrophobic interactions [15]. The 219-270 C-terminal domain is dimeric in solution. It consists of two symmetric monomers of five antiparallel $\beta$-strands, which form a $\beta$-barrel and adopt an SH3-like fold $[30,63]$.

Two-domain structures. The X-ray structure of a twodomain construct, consisting of the $\mathrm{N}$-terminal and
CCD domains (residues 1-212), was determined for the W131D, F139D, F185K triple mutant [91]. The asymmetric unit contains four molecules corresponding to two pairs of monomers related by a non crystallographic two-fold axis. Each dimer has well resolved CCD and N-terminal domains connected by a highly disordered linking region (residues 47-55). The structure of the two dimers differs only slightly in terms of the relative position of the two domains, the dihedral angle between these domains differing by $15^{\circ}$. The structures of individual domains in this model correspond well to those obtained for the isolated CCD and $\mathrm{N}$-terminal domains. The most notable difference concerns the dimer interface between the N-terminal domains and those in the isolated 1-45 domain.

The X-ray structure of the second two-domain construct (residues 52-288), obtained from a highly mutated protein (C56S/W131D/F139D/F185K/C180S), shows a two-fold symmetric dimer [17]. The two domains, the CCD and C-terminal domain, are connected by a perfect helix formed by residues 195 to 221 . The local structure of each domain is similar to that obtained for the isolated domains, but the dimer C-terminal interface differs from that suggested by NMR data for the isolated C-terminal domain.

Catalytic loop structure. The integrity of the 140-149 catalytic loop is required for IN activity, but its exact role in the catalytic reaction remains unclear. Interest in the catalytic loop has recently increased, with the emergence of the Y143R/C, Q148R/K/H and G140S mutations located within this loop and of $\mathrm{N} 155 \mathrm{H}$ mutations in the catalytic site linked to the development of resistance to raltegravir [69]. The conformational flexibility of this loop is believed to be important for the catalytic steps following DNA binding, and decreases in the loop flexibility greatly reduce activity [38]. In most published structures, the structure of the catalytic loop was not well characterized due to its high degree of flexibility. Some published structures include a partially resolved loop, the complete loop being observed only in five structures corresponding to the F185H single mutant, the W131E/F185K double mutant or the G140A/G149A/F185K triple mutant. The conformation of the loop differed between these structures. An in silico study of the structure of the 140-149 loop identified a W-shaped hairpin that can move, as a single body, in a gate-like manner toward the active site - an observation consistent with molecular dynamics simulations [71].

The dynamic behavior of the HIV-1 IN catalytic domain has been described for the wild-type enzyme, the INSTI-resistant T66I/M154I and G140A/G149A mutants and in presence of the 5-CITEP inhibitor [8, 9, $61,62,74]$. These analysis demonstrated that significant conformational change occurs in the active site. However, molecular modeling demonstrated that the two primary pathways of resistance involving residues Q148 and N155 maintained all the structural features of the active site and catalytic loop. By contrast, the specific interactions between the mutated amino acids selected by raltegravir and DNA base pairs differed from those of the wild-type enzyme, accounting for the differences in efficacy between the mutant and 
wild-type integrases in vitro [71]. Together with theoretical studies that have predicted that the Q146, Q148, and N144 residues of the loop form a DNA binding site [29], this result suggest that raltegravir acts by competing with DNA for residues N155 and/or Q148. In order to thwart the inhibitory effect, the virus may have to select mutations that maintain the integrity of IN structure while allowing alternative modes of DNA recognition.

Theoretical models. In the absence of complete and accurate experimental data, computational methods have become a key tool for probing the interactions of integrase with inhibitors and substrates. Fragmented data concerning the structure of HIV-1 IN have been used to construct models to improve our understanding of inhibitor binding to the target. Theoretical models of both the dimer and tetramer states have been constructed. De Luca and coworkers described a dimeric model of the full-length IN/viral DNA complex with two $\mathrm{Mg}^{2+}$ cations in the active site, consistent with cross-linking data indicating that the Q148 and Y143 residues interact with viral DNA $[26,33]$. The molecular docking method has also been used to investigate further the interactions of the HIV-1 IN dimer with viral DNA before the 3 ' processing reaction [45].

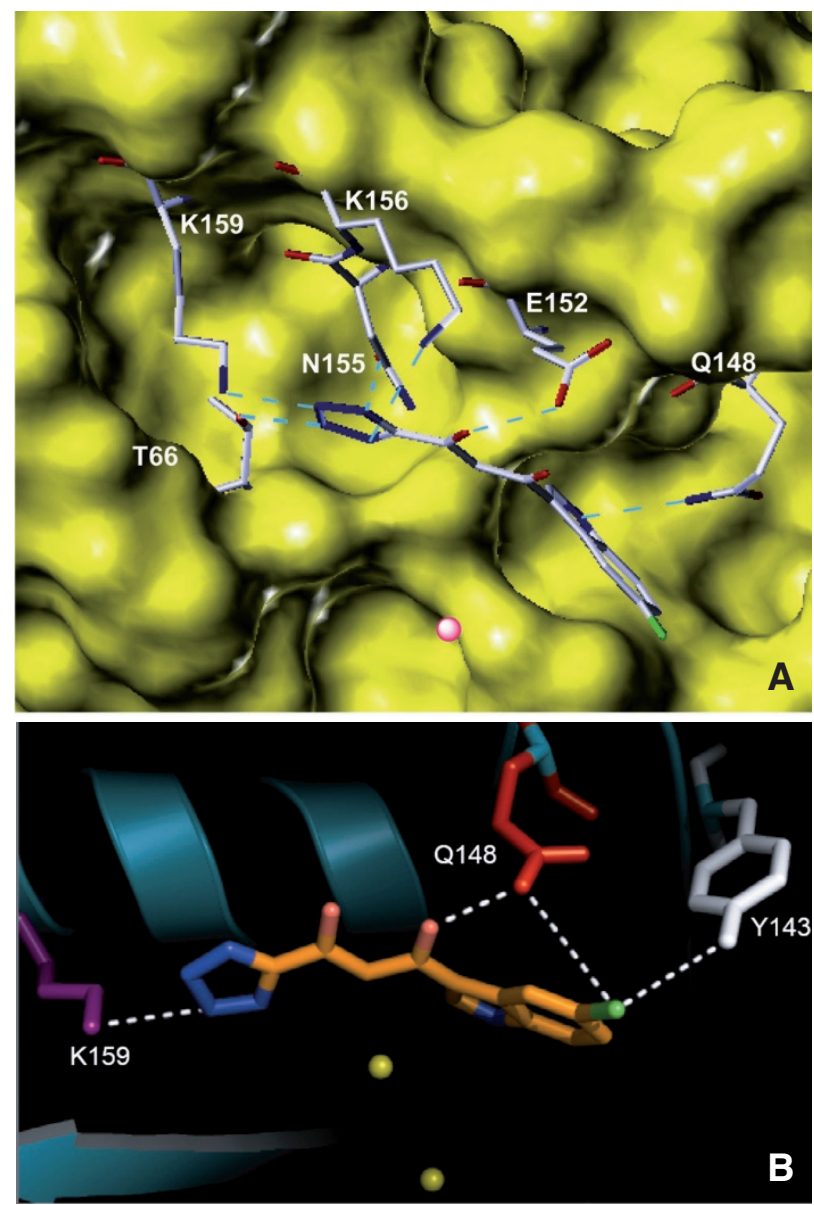

Fig. 3. 5CITEP binding to the HIV-1 integrase. A) X-ray structure of 1QS4 (36). B) In silico modeling (81)(Reproduction authorized). The 5-CITEP inhibitor is shown in similar orientations; $\mathrm{H}$-bonds are indicated by dashed lines, cyan in $\mathrm{A}$ and white in $\mathrm{B}$.
Most theoretical models consider a tetrameric IN alone or in complex with either viral DNA or viral DNA/ target DNA [35 and references therein]. The influence of metal ions on IN•DNA complexes has been explored in a tetramer model constructed by homology modeling and MD simulations [18]. It was found that metal cations could potentially influence the location of the viral DNA on IN. Full-length models of the HIV-1 IN tetramer in complex with both viral and target DNAs have been constructed with either one or two $\mathrm{Mg}^{2+}$ ions in the active site, to ensure consistency with biochemical experimental findings.

Modes of raltegravir interaction with IN. The molecular docking of different DKAs onto the catalytic core domain identified two unique binding areas within the active site, including either the conserved D64D116-E152 motif or the flexible loop region formed by amino acid residues 140-149, and confirmed that the mechanism of inhibition by DKAs involves metal chelation by the $\beta$-ketoenol group [82]. A comparative residue interaction analysis (CoRIA) was recently performed [28], allowing evaluation of the non bonded interaction energies of the inhibitors with individual active site residues and an assessment of the correlation with biological activity, leading to the identification of crucial residues and characterization of interactions between the ligand and receptor. The models suggest that Asp64, Thr66, Val77, Asp116, Glu152 and Lys159 are the key residues influencing the binding of ligands with the integrase. The docking of raltegravir and analogs onto $\mathrm{Mg}^{2+}$-complexed IN demonstrated the establishment of direct interactions between raltegravir and the three catalytic residues D64, D116, and E152, and with residues T66, E92, Y143, Q148, and N155 [84] (Fig. 4, C and Fig. 5, C). This result was again consistent with the findings of clinical experimental resistance profiling and provided a rational for the involvement of E92 and Y143residues in resistance.

A single crystal structure of the IN core domain co-crystallized with an INSTI has been obtained with 5CITEP [36]. The inhibitor is located between the active site residues D64, D116 and E152 (Fig. 3, A). Two $\mathrm{H}$-bonds are formed between the tetrazolium moiety and the K165 and K159 residues involved in DNA binding [50]. The other contacts are the T66 residue implicated in resistance to diketoacids in vitro and the N155, Y143 and Q148 residues involved in raltegravir resistance in vivo. Although obtained in the absence of viral DNA it is assumed that the interactions between 5-CITEP and IN observed in this structure at least partly mimic the contacts between IN and DNA (Fig. $3, \mathrm{~B})$, justifying the use of the integrase CCD•5CITEP complex as a surrogate platform for docking simulations [81]. This model was used to study the mode of binding of raltegravir [64]. Two conformations of raltegravir, differing in the nature of the interacting residues and the method of $\mathrm{Mg}^{2}$ chelation, were obtained (Fig. 4, A and B). However, this compound was systematically located in the vicinity of the Y143, N155 and Q148 residues (Fig. 5, A and B), thereby confirming the role of these three amino acids. 
<smiles></smiles>

A<smiles></smiles>

C<smiles>[M]OC1=C(O[M])C(NCc2ccc(F)cc2)=NC(C(C)(C)C(=O)Nc2nnc(C)o2)C1=O</smiles>

B

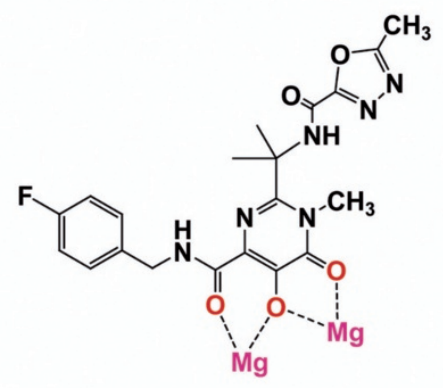

D
Fig. 4. Modeling of $\mathrm{Mg}$ binding by raltegravir. Top: Two different modes of $\mathrm{Mg}$ coordination, A- Mg chelation by oxadiazole-2-carboxylate (in E-conformation) and $\mathrm{B}-\mathrm{Mg}$ chelation by the $\beta$-keto enol group of carbonylamino-1-Nalkyl-5-hydroxypyrimidione (in Z-conformation) (docked on the IN/5-CITEP complex by Autodock 4.0) (64). Bottom: C - Mg chelation by the $\beta$-keto enol group (docked on the 1BL3 crystal structure by GOLD 3.2) (84) and D Two-metal coordination by raltegravir (induced-fit docking on the IN $\cdot$ DNA complex)(5). The chelating centers, $\mathrm{O}$ and $\mathrm{N}$, and $\mathrm{Mg}$ cations are shown in red, blue and magenta, respectively.
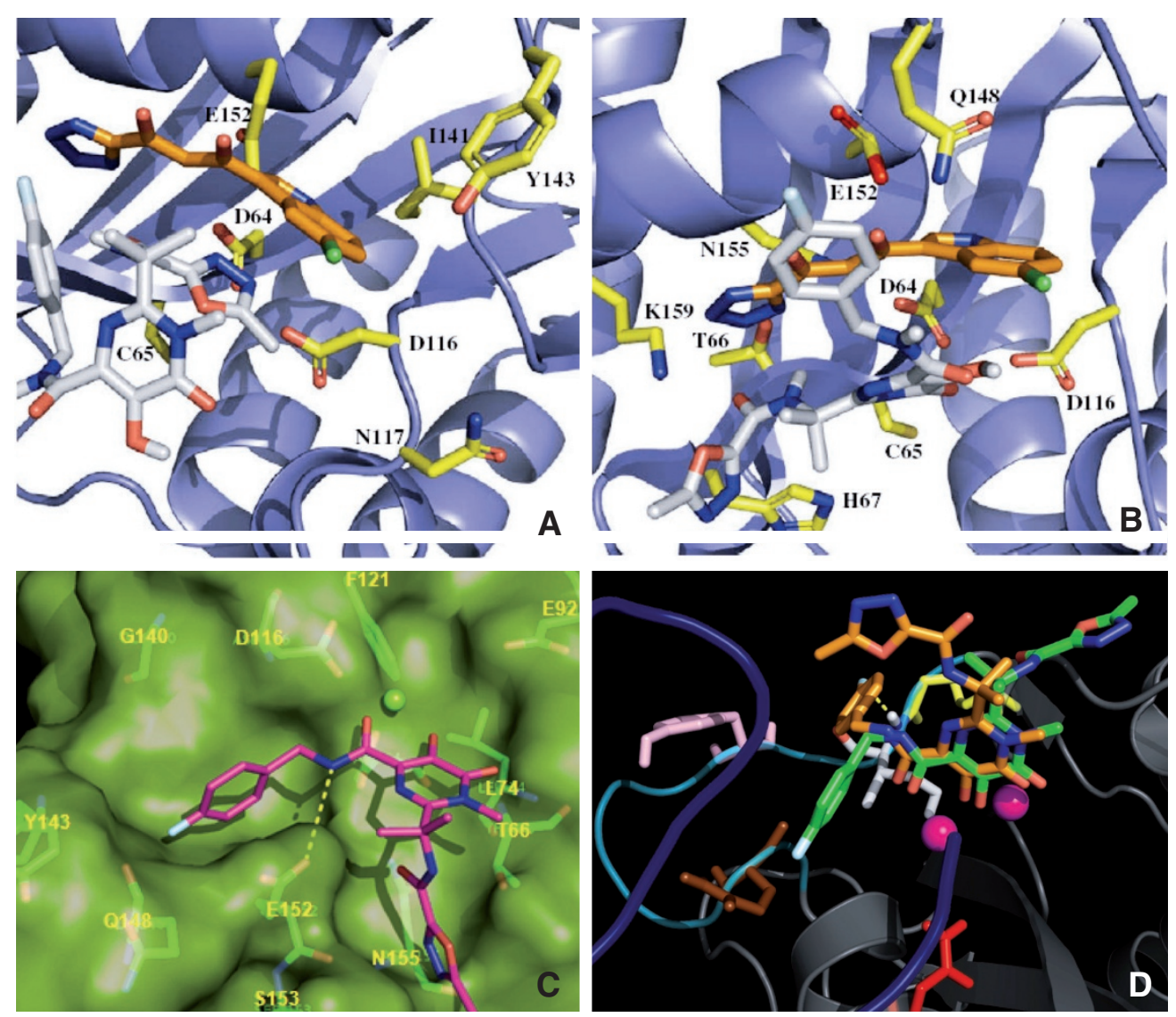

Fig. 5. Raltegravir target binding: A) and B) Two different conformations obtained by inhibitor docking on the 5CITEP•IN complex by Autodock 4.0 (64). Inhibitors and residues in close contact are indicated as sticks and the protein is shown as a secondary structure cartoon. 5CITEP marking the terminal portion of 3'-processed viral DNA, the catalytic triad (D64, D116, E152) and interacting key residues are shown as gray, orange and yellow carbon backbone representations, respectively. C) The best docking (GOLD 3.2) of the inhibitor on the 1BL3 IN crystal structure (84). Inhibitor (magenta) and residues in close contact (green) are indicated as sticks; D) Inhibitor induced-fit docking on the IN•DNA complex (5). Two positions of the inhibitor, 1 (orange) and 2 (green); residues in close contact are indicated as sticks and the protein is shown as a secondary structure cartoon. The images are reproduced from the corresponding papers with kind permission of the authors. 
The contribution of viral DNA has been assessed in models of IN $\bullet$ DNA complexes used for the docking of diverse set of INSTIs. The inhibitors bound close to the three catalytic residues and interacted with the donor DNA. Moreover, these studies confirmed several key observations: the inhibitor binding site exists only after the 3' processing of vDNA and the hydrophobic tail binds in the hydrophobic pocket formed principally by the flexible active site loop [18]. The refinement of this strategy by induced-fit docking (IFD) demonstrated that raltegravir binding involved a twometal mechanism (Fig. 4, D) and close interactions with the terminal adenine of the 3'-processed viral DNA (Fig. 5, D), consistent with the findings of biochemical experiments, [52]. An alternative computational strategy involves the use of the coordinates of the Tn5 transposase-DNA complex as a three-dimensional target for the docking of INSTIs [6]. Finally, the effect of INSTI-resistant mutations has been investigated directly through docking and molecular dynamics simulations of the S-1360 DKA on models of mutant integrases [96]. The presence of mutations resulted in the exclusion of the inhibitor from the DNA binding site.

In conclusion, with the authorization for clinical use of raltegravir and the arrival of other potent new ARVs, the therapeutic management of patients with multi-failure is facilitated with virological success rate up to $90 \%$ in the most favorable case when fully active molecules are associated. Furthermore, in June 2009, Isentress received an extended indication for previously untreated patients, in combination with standard treatment. The chemical and molecular determinants of raltegravir potency are now well understood and the nature of the interactions with its target in the context of the integrase/vDNA complex is beginning to be elucidated owing to the contribution of molecular modeling. This knowledge contributes to our understanding of the molecular reasons for the emergence of the resistance pathways, mainly based on the Q148, N155 and Y143 residues. The mutation of these key residues, involved in the specific interaction of integrase with its DNA substrate, into well-defined amino acids, prevent raltegravir to bind efficiently to integrase whilst maintaining the catalytic activity of the enzyme. Modeling studies suggested that second generation inhibitors should molecules depart from the model of inhibition demonstrated by raltegravir, involving simultaneously metal chelation and interaction with the catalytic loop or risk seeing the emergence of cross-resistance as already demonstrated with elvitegravir.

Acknowledgments: The research of Jean-François Mouscadet's group is funded by the Centre National pour la Recherche Scientifique (CNRS), l'Ecole Normale Supérieure de Cachan (ENSC), l'Agence Nationale de Recherche sur le Sida et les Hépatites (ANRS) and Sidaction.

\section{REFERENCES}

1. Arhel, N. J., S. Souquere-Besse, S. Munier, P. Souque, S. Guadagnini, S. Rutherford, M. C. Prevost, T. D. Allen, and P. Charneau. 2007. HIV-1 DNA Flap formation promotes uncoating of the pre-integration complex at the nuclear pore. EMBO J 26:3025-3037.
2. Asante-Appiah, E., S. H. Seeholzer, and A. M. Skalka. 1998 Structural determinants of metal-induced conformational changes in HIV- 1 integrase. J Biol Chem 273:35078-35087.

3. Asante-Appiah, E., and A. M. Skalka. 1999. HIV-1 integrase: structural organization, conformational changes, and catalysis. Adv. Virus Res. 52:351-369.

4. Barreca, M. L., S. Ferro, A. Rao, L. De Luca, M. Zappala, A. M. Monforte, Z. Debyser, M. Witvrouw, and A. Chimirri. 2005. Pharmacophore-based design of HIV-1 integrase strand-transfer inhibitors. J Med Chem 48:7084-8.

5. Barreca, M. L., N. Iraci, L. De Luca, and A. Chimirri. 2009. Induced-fit docking approach provides insight into the binding mode and mechanism of action of HIV-1 integrase inhibitors. Chem Med Chem. In press. Epub ahead of print 2009/06/23.

6. Barreca, M. L., F. Ortuso, N. Iraci, L. De Luca, S. Alcaro, and A. Chimirri. 2007. Tn5 transposase as a useful platform to simulate HIV-1 integrase inhibitor binding mode. Biochem Biophys Res Commun 363:554-60.

7. Beese, L. S., and T. A. Steitz. 1991. Structural basis for the 3'-5' exonuclease activity of Escherichia-coli DNA polymerase-I - A 2 metal-ion mechanism. EMBO J 10:25-33.

8. Brigo, A., K. W. Lee, F. Fogolari, G. I. Mustata, and J. M. Briggs. 2005. Comparative molecular dynamics simulations of HIV-1 integrase and the T66I/M154I mutant: binding modes and drug resistance to a diketo acid inhibitor. Proteins 59:723-41.

9. Brigo, A., K. W. Lee, G. Iurcu Mustata, and J. M. Briggs. 2005. Comparison of multiple molecular dynamics trajectories calculated for the drug-resistant HIV-1 integrase T66I/M154I catalytic domain. Biophys J 88:3072-82.

10. Brin, E., J. Yi, A. M. Skalka, and J. Leis. 2000. Modeling the late steps in HIV-1 IN-catalyzed DNA integration. J Biol Chem. 275: 39287-95

11. Brown, P. O. 1990. Integration of retroviral DNA. Curr Top Microbiol Immunol. 157:19-48:19-48.

12. Brussel, A., and P. Sonigo. 2004. Evidence for gene expression by unintegrated human immunodeficiency virus type 1 DNA species. J Virol 78:11263-71.

13. Bujacz, G., J. Alexandratos, A. Wlodawer, G. Merkel, M. Andrake, R. A. Katz, and A. M. Skalka. 1997. Binding of different divalent cations to the active site of avian sarcoma virus integrase and their effects on enzymatic activity. J Biol Chem. 272:18161-18168.

14. Bushman, F. D., and R. Craigie. 1991. Activities of human immunodeficiency virus (HIV) integration protein in vitro: specific cleavage and integration of HIV DNA. Proc. Natl. Acad. Sci. USA. 88:1339-1343.

15. Cai, M., R. Zheng, M. Caffrey, R. Craigie, G. M. Clore, and A. M. Gronenborn. 1997. Solution structure of the N-terminal zinc binding domain of HIV-1 integrase. Nat. Struct. Biol. 4:567-577.

16. Ceccherini-Silberstein, F., I. Malet, R. D'Arrigo, A. Antinori, A. G. Marcelin, and C. F. Perno. 2009. Characterization and structural analysis of HIV-1 integrase conservation. AIDS Rev 11:17-29.

17. Chen, J. C., J. Krucinski, L. J. Miercke, J. S. Finer-Moore, A. H. Tang, A. D. Leavitt, and R. M. Stroud. 2000. Crystal structure of the HIV-1 integrase catalytic core and C-terminal domains: a model for viral DNA binding. Proc. Natl. Acad. Sci. USA 97:8233-8238.

18. Chen, X., M. Tsiang, F. Yu, M. Hung, G. S. Jones, A. Zeynalzadegan, X. Qi, H. Jin, C. U. Kim, S. Swaminathan, and J. M. Chen. 2008. Modeling, analysis, and validation of a novel HIV integrase structure provide insights into the binding modes of potent integrase inhibitors. J Mol Biol 380: 504-19.

19. Chirch, L. M., S. Morrison, and R. T. Steigbigel. 2009. Treatment of HIV infection with raltegravir. Expert Opin Pharmacother 10:1203-11.

20. Chiu, T. K., and D. R. Davies. 2004. Structure and function of HIV-1 integrase. Curr. Top. Med. Chem. 4:965-977.

21. Chow, S. A., K. A. Vincent, V. Ellison, and P. O. Brown. 1992. Reversal of integration and DNA splicing mediated by 
integrase of human immunodeficiency virus. Science 255 723-726.

22. Chun, T. W., L. Stuyver, S. B. Mizell, L. A. Ehler, J. A. Mican, M. Baseler, A. L. Lloyd, M. A. Nowak, and A. S. Fauci. 1997. Presence of an inducible HIV-1 latent reservoir during highly active antiretroviral therapy. Proc Natl Acad Sci USA 94:13193-13197.

23. Cocohoba, J., and B. J. Dong. 2008. Raltegravir: the first HIV integrase inhibitor. Clin Ther 30:1747-65.

24. Cooper, D. A., R. T. Steigbigel, J. M. Gatell, J. K. Rockstroh, C. Katlama, P. Yeni, A. Lazzarin, B. Clotet, P. N. Kumar, J. E. Eron, M. Schechter, M. Markowitz, M. R. Loutfy, J. L. Lennox, J. Zhao, J. Chen, D. M. Ryan, R. R. Rhodes, J. A. Killar, L. R. Gilde, K. M. Strohmaier, A. R. Meibohm, M. D. Miller, D. J. Hazuda, M. L. Nessly, M. J. DiNubile, R. D. Isaacs, H. Teppler, B. Y. Nguyen, and B. S. Teams. 2008. Subgroup and resistance analyses of raltegravir for resistant HIV-1 infection. New England Journal of Medicine 359:355-365.

25. Croxtall, J. D., and S. J. Keam. 2009. Raltegravir: a review of its use in the management of HIV infection in treatment-experienced patients. Drugs 69:1059-75.

26. De Luca, L., A. Pedretti, G. Vistoli, M. L. Barreca, L. Villa, P. Monforte, and A. Chimirri. 2003. Analysis of the fulllength integrase-DNA complex by a modified approach for DNA docking. Biochem Biophys Res Commun 310:1083-8.

27. Delelis, O., I. Malet, L. Na, L. Tchertanov, V. Calvez, A. G. Marcelin, F. Subra, E. Deprez, and J. F. Mouscadet. 2009. The G140S mutation in HIV integrases from raltegravir-resistant patients rescues catalytic defect due to the resistance Q148H mutation. Nucleic Acids Res 37:1193-201.

28. Dhaked, D. K., J. Verma, A. Saran, and E. C. Coutinho. 2009. Exploring the binding of HIV-1 integrase inhibitors by comparative residue interaction analysis (CoRIA). J Mol Model 15:233-45

29. Dolan, J., A. Chen, I. T. Weber, R. W. Harrison, and J. Leis. 2009. Defining the DNA substrate binding sites on HIV-1 integrase. J Mol Biol 385:568-79.

30. Eijkelenboom, A. P., R. A. Lutzke, R. Boelens, R. H. Plasterk, R. Kaptein, and K. Hard. 1995. The DNA-binding domain of HIV-1 integrase has an SH3-like fold. Nat Struct Biol 2:807-810.

31. Engelman, A., F. D. Bushman, and R. Craigie. 1993. Identification of discrete functional domains of HIV-1 integrase and their organization within an active multimeric complex. EMBO J 12:3269-3275.

32. Espeseth, A. S., P. Felock, A. Wolfe, M. Witmer, J. Grobler, N. Anthony, M. Egbertson, J. Y. Melamed, S. Young, T. Hamill, J. L. Cole, and D. J. Hazuda. 2000. HIV-1 integrase inhibitors that compete with the target DNA substrate define a unique strand transfer conformation for integrase. Proc Natl Acad Sci USA 97:11244-11249.

33. Esposito, D., and R. Craigie. 1998. Sequence specificity of viral end DNA binding by HIV-1 integrase reveals critical regions for protein-DNA interaction. EMBO J. 17:58325843.

34. Faure, A., C. Calmels, C. Desjobert, M. Castroviejo, A. Caumont-Sarcos, L. Tarrago-Litvak, S. Litvak, and V. Parissi. 2005. HIV-1 integrase crosslinked oligomers are active in vitro. Nucleic Acids Res. 33:977-986.

35. Fenollar-Ferrer, C., V. Carnevale, S. Raugei, and P. Carloni. 2008. HIV-1 integrase-DNA interactions investigated by molecular modelling. Computational and Mathematical Methods in Medicine 9:231-243.

36. Goldgur, Y., R. Craigie, G. H. Cohen, T. Fujiwara, T. Yoshinaga, T. Fujishita, H. Sugimoto, T. Endo, H. Murai, and D. R. Davies. 1999. Structure of the HIV-1 integrase catalytic domain complexed with an inhibitor: a platform for antiviral drug design. Proc Natl Acad Sci USA 96:13040-13043.

37. Goldgur, Y., F. Dyda, A. B. Hickman, T. M. Jenkins, R. Craigie, and D. R. Davies. 1998. Three new structures of the core domain of HIV-1 integrase: an active site that binds magnesium. Proc Natl Acad Sci USA 95:9150-9154.

38. Greenwald, J., V. Le, S. L. Butler, F. D. Bushman, and S.
Choe. 1999. The mobility of an HIV-1 integrase active site loop is correlated with catalytic activity. Biochemistry 38:8892-8898.

39. Grinsztejn, B., B. Y. Nguyen, C. Katlama, J. M. Gatell, A. Lazzarin, D. Vittecoq, C. J. Gonzalez, J. Chen, C. M. Harvey, and R. D. Isaacs. 2007. Safety and efficacy of the HIV-1 integrase inhibitor raltegravir (MK-0518) in treatment-experienced patients with multidrug-resistant virus: a phase II randomised controlled trial. Lancet 369:1261-1269.

40. Grobler, J. A., K. Stillmock, B. Hu, M. Witmer, P. Felock, A. S. Espeseth, A. Wolfe, M. Egbertson, M. Bourgeois, J. Melamed, J. S. Wai, S. Young, J. Vacca, and D. J. Hazuda. 2002. Diketo acid inhibitor mechanism and HIV-1 integrase: implications for metal binding in the active site of phosphotransferase enzymes. Proc Natl Acad Sci USA 99:6661-6666.

41. Guiot, E., K. Carayon, O. Delelis, F. Simon, P. Tauc, E. Zubin, M. Gottikh, J. F. Mouscadet, J. C. Brochon, and E. Deprez. 2006. Relationship between the oligomeric status of HIV-1 integrase on DNA and enzymatic activity. J Biol Chem 281:22707-19.

42. Hazuda, D., M. Iwamoto, and L. Wenning. 2009. Emerging pharmacology: inhibitors of human immunodeficiency virus integration. Annu Rev Pharmacol Toxicol 49:377-94.

43. Hazuda, D. J., P. Felock, M. Witmer, A. Wolfe, K. Stillmock, J. A. Grobler, A. Espeseth, L. Gabryelski, W. Schleif, C. Blau, and M. D. Miller. 2000. Inhibitors of strand transfer that prevent integration and inhibit HIV-1 replication in cells. Science 287:646-650

44. Hazuda, D. J., S. D. Young, J. P. Guare, N. J. Anthony, R. P. Gomez, J. S. Wai, J. P. Vacca, L. Handt, S. L. Motzel, H. J. Klein, G. Dornadula, R. M. Danovich, M. V. Witmer, K. A. Wilson, L. Tussey, W. A. Schleif, L. S. Gabryelski, L. Jin, M. D. Miller, D. R. Casimiro, E. A. Emini, and J. W. Shiver. 2004. Integrase inhibitors and cellular immunity suppress retroviral replication in Rhesus macaques. Science.

45. Hu, J. P., X. Q. Gong, J. G. Su, W. Z. Chen, and C. X. Wang. 2008. Study on the molecular mechanism of inhibiting HIV-1 integrase by EBR28 peptide via molecular modeling approach. Biophys Chem 132:69-80.

46. Iwamoto, M., K. Kassahun, M. D. Troyer, W. D. Hanley, P. Lu, A. Rhoton, A. S. Petry, K. Ghosh, E. Mangin, E. P. DeNoia, L. A. Wenning, J. A. Stone, K. M. Gottesdiener, and J. A. Wagner. 2008. Lack of a pharmacokinetic effect of raltegravir on midazolam: in vitro/in vivo correlation. J Clin Pharmacol 48:209-14.

47. Iwamoto, M., L. A. Wenning, G. C. Mistry, A. S. Petry, S. Y. Liou, K. Ghosh, S. Breidinger, N. Azrolan, M. J. Gutierrez, W. E. Bridson, J. A. Stone, K. M. Gottesdiener, and J. A. Wagner. 2008. Atazanavir modestly increases plasma levels of raltegravir in healthy subjects. Clin Infect Dis 47:137-40.

48. Iwamoto, M., L. A. Wenning, A. S. Petry, M. Laethem, M. De Smet, J. T. Kost, S. A. Merschman, K. M. Strohmaier, S. Ramael, K. C. Lasseter, J. A. Stone, K. M. Gottesdiener, and J. A. Wagner. 2008. Safety, tolerability, and pharmacokinetics of raltegravir after single and multiple doses in healthy subjects. Clin Pharmacol Ther 83:293-9.

49. Jaskolski, M., J. N. Alexandratos, G. Bujacz, and A. Wlodawer. 2009. Piecing together the structure of retroviral integrase, an important target in AIDS therapy. FEBS J 276:2926-46

50. Jenkins, T. M., D. Esposito, A. Engelman, and R. Craigie. 1997. Critical contacts between HIV-1 integrase and viral DNA identified by structure-based analysis and photocrosslinking. EMBO J. 16:6849-6859.

51. Jenkins, T. M., A. B. Hickman, F. Dyda, R. Ghirlando, D. R. Davies, and R. Craigie. 1995. Catalytic domain of human immunodeficiency virus type 1 integrase: identification of a soluble mutant by systematic replacement of hydrophobic residues. Proc Natl Acad Sci USA 92:6057-6061.

52. Johnson, A. A., C. Marchand, S. S. Patil, R. Costi, S. R. Di, T. R. Burke, Jr., and Y. Pommier. 2007. Probing HIV-1 integrase inhibitor binding sites with position-specific integraseDNA cross-linking assays. Mol Pharmacol 71:893-901.

53. Kawasuji, T., M. Fuji, T. Yoshinaga, A. Sato, T. Fujiwara, 
and R. Kiyama. 2006. A platform for designing HIV integrase inhibitors. Part 2: a two-metal binding model as a potential mechanism of HIV integrase inhibitors. Bioorg Med Chem 14:8420-9.

54. Kilzer, J. M., T. Stracker, B. Beitzel, K. Meek, M. Weitzman, and F. D. Bushman. 2003. Roles of host cell factors in circularization of retroviral DNA. Virology 314:460-467.

55. Lee, M. S., and R. Craigie. 1994. Protection of retroviral DNA from autointegration: involvement of a cellular factor. Proc Natl Acad Sci USA 91:9823-9827.

56. Lee, S. P., and M. K. Han. 1996. Zinc stimulates $\mathrm{Mg}^{2+}$-dependent 3 '-processing activity of human immunodeficiency virus type 1 integrase in vitro. Biochemistry 35:3837-3844.

57. Leh, H., P. Brodin, J. Bischerour, E. Deprez, P. Tauc, J. C. Brochon, E. LeCam, D. Coulaud, C. Auclair, and J. F. Mouscadet. 2000. Determinants of $\mathrm{Mg}^{2+}$-dependent activities of recombinant human immunodeficiency virus type 1 integrase. Biochemistry 39:9285-9294.

58. Lennox, J. L., E. Dejesus, A. Lazzarin, R. B. Pollard, J. V. Madruga, D. S. Berger, J. Zhao, X. Xu, A. Williams-Diaz, A. J. Rodgers, R. J. Barnard, M. D. Miller, M. J. Dinubile, B. Y. Nguyen, R. Leavitt, and P. Sklar. 2009. Safety and efficacy of raltegravir-based versus efavirenz-based combination therapy in treatment-naive patients with HIV-1 infection: a multicentre, double-blind randomised controlled trial. Lancet. In press. Epub ahead of print 2009/08/04.

59. Li, L., J. M. Olvera, K. E. Yoder, R. S. Mitchell, S. L. Butler, M. Lieber, S. L. Martin, and F. D. Bushman. 2001. Role of the non-homologous DNA end joining pathway in the early steps of retroviral infection. EMBO J. 20:3272-3281.

60. Li, M., and R. Craigie. 2005. Processing of viral DNA ends channels the HIV-1 integration reaction to concerted integration. J.Biol.Chem. 280:29334-29339.

61. Lins, R. D., A. Adesokan, T. A. Soares, and J. M. Briggs. 2000. Investigations on human immunodeficiency virus type 1 integrase/DNA binding interactions via molecular dynamics and electrostatics calculations. Pharmacol Ther 85:123131.

62. Lins, R. D., J. M. Briggs, T. P. Straatsma, H. A. Carlson, J. Greenwald, S. Choe, and J. A. McCammon. 1999. Molecular dynamics studies on the HIV-1 integrase catalytic domain. Biophys J 76:2999-3011.

63. Lodi, P. J., J. A. Ernst, J. Kuszewski, A. B. Hickman, A. Engelman, R. Craigie, G. M. Clore, and A. M. Gronenborn. 1995. Solution structure of the DNA binding domain of HIV-1 integrase. Biochemistry 34:9826-9833.

64. Loizidou, E. Z., C. D. Zeinalipour-Yazdi, T. Christofides, and L. G. Kostrikis. 2009. Analysis of binding parameters of HIV-1 integrase inhibitors: correlates of drug inhibition and resistance. Bioorg Med Chem 17:4806-18.

65. Lovell, S., I. Y. Goryshin, W. R. Reznikoff, and I. Rayment. 2002. Two-metal active site binding of a Tn5 transposase synaptic complex. Nat Struct Biol 9:278-81.

66. Maiga, A. I., I. Malet, C. Soulie, A. Derache, V. Koita, B. Amellal, L. Tchertanov, O. Delelis, L. Morand-Joubert, J. F. Mouscadet, R. Murphy, M. Cisse, C. Katlama, V. Calvez, and A. G. Marcelin. 2009. Genetic barriers for integrase in hibitor drug resistance in HIV type-1 B and CRF02_AG subtypes. Antivir Ther 14:123-9.

67. Maignan, S., J. P. Guilloteau, Q. Zhou-Liu, C. Clement-mella, and V. Mikol. 1998. Crystal structures of the catalytic domain of HIV-1 integrase free and complexed with its metal cofactor: high level of similarity of the active site with other viral integrases. J.Mol.Biol. 282:359-368.

68. Malet, I., O. Delelis, C. Soulie, M. Wirden, L. Tchertanov, P. Mottaz, G. Peytavin, C. Katlama, J. F. Mouscadet, V. Calvez, and A. G. Marcelin. 2009. Quasispecies variant dynamics during emergence of resistance to raltegravir in HIV-1-infected patients. J Antimicrob Chemother 63:795804.

69. Malet, I., O. Delelis, M. A. Valantin, B. Montes, C. Soulie, M. Wirden, L. Tchertanov, G. Peytavin, J. Reynes, J. F. Mouscadet, C. Katlama, V. Calvez, and A. G. Marcelin. 2008. Mutations associated with failure of raltegravir treat- ment affect integrase sensitivity to the inhibitor in vitro. Antimicrob Agents Chemother. 52:1351-1358.

70. Miller, M., M. Witmer, K. Stillmock, P. Felock, L. Ecto, J. Flynn, W. Schleif, G. Dornadula, R. Danovich, and D. Hazuda. 2006. Biochemical and antiviral activity of MK0518, a potent HIV integrase inhibitor. AIDS 2006 - XVI International AIDS Conference Abstract no. THAA0302.

71. Mouscadet, J. F., R. Arora, J. Andre, J. C. Lambry, O. Delelis, I. Malet, A. G. Marcelin, V. Calvez, and L. Tchertanov. 2009. HIV-1 IN alternative molecular recognition of DNA induced by raltegravir resistance mutations. J Mol Recognit. Epub ahead of print 2009/07/23

72. Murray, J. M., S. Emery, A. D. Kelleher, M. Law, J. Chen, D. J. Hazuda, B. Y. Nguyen, H. Teppler, and D. A. Cooper. 2007. Antiretroviral therapy with the integrase inhibitor raltegravir alters decay kinetics of HIV, significantly reducing the second phase. AIDS 21:2315-21.

73. Nakajima, N., R. Lu, and A. Engelman. 2001. Human immunodeficiency virus type 1 replication in the absence of integrase-mediated dna recombination: definition of permissive and nonpermissive T-cell lines. J Virol 75:7944-7955.

74. Nunthaboot, N., S. Pianwanit, V. Parasuk, J. O. Ebalunode, J. M. Briggs, and S. Kokpol. 2007. Hybrid quantum mechani$\mathrm{cal} /$ molecular mechanical molecular dynamics simulations of HIV-1 integrase/inhibitor complexes. Biophys J 93:3613-26.

75. O'Brien, W. A., A. Namazi, H. Kalhor, S. H. Mao, J. A. Zack, and I. S. Chen. 1994. Kinetics of human immunodeficiency virus type 1 reverse transcription in blood mononuclear phagocytes are slowed by limitations of nucleotide precursors. J Virol 68:1258-1263.

76. Pace, P., M. E. Di Francesco, C. Gardelli, S. Harper, E. Muraglia, E. Nizi, F. Orvieto, A. Petrocchi, M. Poma, M. Rowley, R. Scarpelli, R. Laufer, O. Gonzalez Paz, E. Monteagudo, F. Bonelli, D. Hazuda, K. A. Stillmock, and V. Summa. 2007. Dihydroxypyrimidine-4-carboxamides as novel potent and selective HIV integrase inhibitors. J Med Chem 50:2225-39.

77. Pauza, C. D., P. Trivedi, T. S. McKechnie, D. D. Richman, and F. M. Graziano. 1994. 2-LTR circular viral DNA as a marker for human immunodeficiency virus type 1 infection in vivo. Virology 205:470-478.

78. Pommier, Y., A. A. Johnson, and C. Marchand. 2005. Integrase inhibitors to treat HIV/AIDS. Nat Rev Drug Discov 4:236-248

79. Roquebert, B., F. Damond, G. Collin, S. Matheron, G. Peytavin, A. Benard, P. Campa, G. Chene, F. Brun-Vezinet, and D. Descamps. 2008. HIV-2 integrase gene polymorphism and phenotypic susceptibility of HIV-2 clinical isolates to the integrase inhibitors raltegravir and elvitegravir in vitro. J Antimicrob Chemother 62:914-20.

80. Sato, M., T. Motomura, H. Aramaki, T. Matsuda, M. Yamashita, Y. Ito, H. Kawakami, Y. Matsuzaki, W. Watanabe, K. Yamataka, S. Ikeda, E. Kodama, M. Matsuoka, and H. Shinkai. 2006. Novel HIV-1 integrase inhibitors derived from quinolone antibiotics. J Med Chem 49:1506-8.

81. Savarino, A., M. Pistello, D. D'Ostilio, E. Zabogli, F. Taglia, F. Mancini, S. Ferro, D. Matteucci, L. De Luca, M. L. Barreca, A. Ciervo, A. Chimirri, M. Ciccozzi, and M. Bendinelli. 2007. Human immunodeficiency virus integrase inhibitors efficiently suppress feline immunodeficiency virus replication in vitro and provide a rationale to redesign antiretroviral treatment for feline AIDS. Retrovirology 4:79.

82. Sechi, M., A. Bacchi, M. Carcelli, C. Compari, E. Duce, E. Fisicaro, D. Rogolino, P. Gates, M. Derudas, L. Q. Al-Mawsawi, and N. Neamati. 2006. From ligand to complexes: inhibition of human immunodeficiency virus type 1 integrase by beta-diketo acid metal complexes. Journal of Medicinal Chemistry 49:4248-4260.

83. Semenova, E. A., C. Marchand, and Y. Pommier. 2008. HIV-1 integrase inhibitors: update and perspectives. Adv Pharmacol 56:199-228.

84. Serrao, E., S. Odde, K. Ramkumar, and N. Neamati. 2009. Raltegravir, elvitegravir, and metoogravir: the birth of "metoo" HIV-1 integrase inhibitors. Retrovirology 6:25. 
85. Sichtig, N., S. Sierra, R. Kaiser, M. Daumer, S. Reuter, E. Schulter, A. Altmann, G. Fatkenheuer, U. Dittmer, H. Pfister, and S. Esser. 2009. Evolution of raltegravir resistance during therapy. J Antimicrob Chemother 64:25-32.

86. Smolov, M., M. Gottikh, V. Tashlitskii, S. Korolev, I. Demidyuk, J. C. Brochon, J. F. Mouscadet, and E. Deprez. 2006. Kinetic study of the HIV-1 DNA 3'-end processing. FEBS J. 273:1137-1151.

87. Spina, C. A., J. C. Guatelli, and D. D. Richman. 1995. Establishment of a stable, inducible form of human immunodeficiency virus type $1 \mathrm{DNA}$ in quiescent CD4 lymphocytes in vitro. J Virol 69:2977-88.

88. Steigbigel, R. T., D. A. Cooper, P. N. Kumar, J. E. Eron, M. Schechter, M. Markowitz, M. R. Loutfy, J. L. Lennox, J. M. Gatell, J. K. Rockstroh, C. Katlama, P. Yeni, A. Lazzarin, B. Clotet, J. Zhao, J. Chen, D. M. Ryan, R. R. Rhodes, J. A. Killar, L. R. Gilde, K. M. Strohmaier, A. R. Meibohm, M. D. Miller, D. J. Hazuda, M. L. Nessly, M. J. DiNubile, R. D. Isaacs, B. Nguyen, H. Teppler, and B. S. Teams. 2008. Raltegravir with optimized background therapy for resistant HIV-1 infection. New England Journal of Medicine 359:339-354

89. Summa, V., A. Petrocchi, F. Bonelli, B. Crescenzi, M. Donghi, M. Ferrara, F. Fiore, C. Gardelli, O. Gonzalez Paz, D. J. Hazuda, P. Jones, O. Kinzel, R. Laufer, E. Monteagudo, E. Muraglia, E. Nizi, F. Orvieto, P. Pace, G. Pescatore, R. Scarpelli, K. Stillmock, M. V. Witmer, and M. Rowley. 2008. Discovery of raltegravir, a potent, selective orally bioavailable HIV-integrase inhibitor for the treatment of HIV-AIDS infection. J Med Chem 51:5843-55.

90. Tchertanov, L., and J. F. Mouscadet. 2007. Target recognition by catechols and beta-ketoenols: potential contribution of hydrogen bonding and $\mathrm{Mn} / \mathrm{Mg}$ chelation to HIV-1 integrase inhibition. J Med Chem 50:1133-1145.

91. Wang, J. Y., H. Ling, W. Yang, and R. Craigie. 2001. Structure of a two-domain fragment of HIV-1 integrase: implications for domain organization in the intact protein. EMBO J 20:7333-7343

92. Wu, Y., and J. W. Marsh. 2001. Selective transcription and modulation of resting $\mathrm{T}$ cell activity by preintegrated HIV DNA. Science 293:1503-1506.
93. Zack, J. A. 1995. The role of the cell cycle in HIV-1 infection. Adv Exp Med Biol 374:27-31.

94. Zack, J. A., A. M. Haislip, P. Krogstad, and I. S. Chen. 1992. Incompletely reverse-transcribed human immunodeficiency virus type 1 genomes in quiescent cells can function as intermediates in the retroviral life cycle. J.Virol. 66:1717-1725.

95. Zamborlini, A., J. Lehmann-Che, E. Clave, M. L. Giron, J. Tobaly-Tapiero, P. Roingeard, S. Emiliani, A. Toubert, H. de The, and A. Saib. 2007. Centrosomal pre-integration latency of HIV-1 in quiescent cells. Retrovirology 4:63.

96. Zhang, X. Y., H. Q. He, B. Liu, and C. X. Wang. 2009. A study on drug resistance mechanism of HIV-1 integrase mutants by molecular modeling. Progress in Biochemistry and Biophysics 36:592-600.

97. Zhou, Y., H. Zhang, J. D. Siliciano, and R. F. Siliciano. 2005. Kinetics of human immunodeficiency virus type 1 decay following entry into resting CD4+ $\mathrm{T}$ cells. J Virol 79:2199-210.

98. Zhuang, L., J. S. Wai, M. W. Embrey, T. E. Fisher, M. S. Egbertson, L. S. Payne, J. P. Guare, Jr., J. P. Vacca, D. J. Hazuda, P. J. Felock, A. L. Wolfe, K. A. Stillmock, M. V. Witmer, G. Moyer, W. A. Schleif, L. J. Gabryelski, Y. M. Leonard, J. J. Lynch, Jr., S. R. Michelson, and S. D. Young. 2003. Design and synthesis of 8-hydroxy-[1,6]naphthyridines as novel inhibitors of HIV-1 integrase in vitro and in infected cells. J Med Chem 46:453-456.

Address for correspondence:

Jean-François Mouscadet

LBPA, CNRS UMR8113

Ecole Normale Supérieure de Cachan

61 avenue du Président Wilson

94235 Cachan cedex France

Tel.: $\quad+33(0) 147407675$

Fax: $\quad+33(0) 147407671$

E-mail: mouscadet@lbpa.ens-cachan.fr 